\title{
A Study on the Effect of Fatty Acids on Nutrition.
}

\author{
II.-Experiments with Diets composed of Rice, Oil and \\ Lipoid containing Linoleic or Linolenic Acid.
}

By

Ume TANGE.

(Received August 23, 1933.)

When young rats were restricted to fat-free diets, they developed characteristic symptoms accompanying impairment of growth, denuded areas on skin and "scaly" condition of feet, and they were cured by the administration of either linoleic or linolenic acid ${ }^{(1)}$. This observation led the author to further determination as to what function oils and phospholipins containing these essential acids play in animal physiology.

Recently Evans and Lepkovsky ${ }^{(2)}$ indicated that the fatty acids from rice-starch were very potent in relieving disease in rats fed on a fat-free diet, while the fatty materials from potato-starch were ineffective. In our experiments, however, when potato-starch in the fat-free diet was replaced by either polished-rice powder or rice-starch, the growth of the animals was arrested and the fur was stained with blood, which was supposed to be bled around the nose and mouth. In this case, unfortunately, the sick animals were not cured with either linoleic or linolenic acid even though a high level of the yeast extract was administered, but the substitution of whole dried yeast resulted in a marked growth, recovering the ill conditions. This evidence suggested, therefore, the possibility of the presence in the yeast of other growth factors, not present in the yeast extract. ${ }^{(3)(4)}$

\section{Experimental.}

Preparation of Mlaterials used in the Fxperiments.

a) Lecithin :- Purification of lecithin from "Soyalex".*

Two hundred grams of "Soyalex" were extracted with ether at room temperatur. Into the extract two volumes of pure acetone were added, and allowed to stand until a precipitate had settled out: This precipitated the phospholipins and left most of the fat and cholesterol in solution. The clear solution was then decanted from the precipitate, redissolved in ether and

* "Soyalex" (the crude lecithin prepared from soy-bean by the alcohol extraction method in the Central Institute of the South Manchurian Railway Company) was kindly furnished to the author by the Onzeki Company in Tokyo. 
reprecipitated, and this was repeated until the ethereal solution was entirely clear. By the addition now of three volumes of absolute alcohol to each volume of ether, nearly all of the cephalin was precipitated, but lecithin remained in solution. Into the filtrate a warm solution of $\mathrm{CdCl}_{2}$ in $85 \%$ alcohol was added until no more precipitate formed. The lecithin-C $\mathrm{CCl}_{2}$. precipitated out as white crystals. This was washed several, times with absolute alcohol until the filtrate had become colourless. The lecithin- $\mathrm{CdCl}_{2}$ was now suspended in $95 \%$ alcohol and decomposed with alcoholic saturated solution of $\left(\mathrm{NH}_{4}\right)_{2} \mathrm{CO}_{3}$. The filtrate was then evaporated under reduced pressure in $\mathrm{CO}_{2}$ atmosphere; and the residue was treated with ether to remove the admixture of $\left(\mathrm{NH}_{4}\right)_{3} \mathrm{CO}_{3}$ and $\mathrm{Cd}$ salts. $\mathrm{By}$ the addition of acetone into the ether extract the lecithin precipitated out as a pale-yellow waxy substance: This was washed several times with acetone and dried in vacuum. About $40 \mathrm{~g}$ of lecithin were obtained, which had an jodine value of 75 (Wijs). The analysis of total N (Kjeldahl): $2.00 \%$ and $\mathrm{P}$ (molybdate-method): 4.31\%, N/P: 1.08/1.00.

Separation of saturated and unsaturated fatty acids in lecithin.

Forty grams of lecithin obtained by the method mentioned above were heated on a water bath for $2 \sim 3$ hours with saturated $\mathrm{Ba}(\mathrm{OH})$, solution, and the resulted $\mathrm{Ba}$ soaps were now decomposed with $10 \% \mathrm{HCl}$. By extracting the hydrolyzed liquid with ether, about $20 \mathrm{~g}$ of fatty acids were obtained, which had an iodine value of 108 (Wijs). The mixed acids were converted into lead salts by the usual method; the lead salts of the unsaturated fatty acids were removed by thorough extraction with ether. After separating the ether-soluble lead salts from the insoluble ones, each of them was hydrolyzed in ether medium with $\mathrm{HCl}$. Thus, $5.0 \mathrm{~g}$ of saturated acids having $\mathrm{mp} 56 \sim$ $57^{\circ} \mathrm{C}$ and $7.5 \mathrm{~g}$ of unsaturated acids having an iodine value of 130 (Wijs) were obtained.

b) Preparation of vitamin $B_{2}:-$ The most potent concentrate of vitamin $B_{2}$ recorded in the literature seems to be that obtained by Narayanan and Drummond $^{(\sigma)}$ by means of adsorption by fuller's earth from yeast extract of pH 0.1 .

Two hundred grams of the baker's yeast powder were treated in like manner as described in a previous paper, (1) except with alcohol of $50 \%$ concentration. After extracting the concentrated yeast extract with ether to remove fat completely, a saturated solution of $\mathrm{Pb}\left(\mathrm{C}_{2} \mathrm{H}_{3} \mathrm{O}_{2}\right)_{2}$ was added into it. The lead acetate precipitate was decomposed by suspending it in warm water and slowly adding $10 \% \mathrm{H}_{2} \mathrm{SO}_{4}$ with constant stirring until acid to congo-red, whereupon the lead sulphate was removed by filtration. In order to remove any trace of lead that might be present, $\mathrm{H}_{2} \mathrm{~S}$ was passed through the filtrate 
to saturation and the whole allowed to stand for several hours. The reaction of the filtrate was then rendered approximately $0.9 \mathrm{~N}$ with $\mathrm{H}_{2} \mathrm{SO}_{4}$, being almost at $\mathrm{pH} 0.1$, and treated with $3 \mathrm{~g}$ of fuller's earth for every $100 \mathrm{c.c}$ of the filtrate.

The mixture was well stirred mechanically for $1 / 2$ hour, then filtered and the earth was well washed with $0.9 \mathrm{~N} \mathrm{H}_{2} \mathrm{SO}_{4}$. The filtrate and washing were similarly treated, a second time with $1.5 \mathrm{~g}$ of fuller's earth for each 100 c.c.

Thus, $35 \mathrm{~g}$ of the "activated" fuller's earth were obtained, adsorbing nearly $2.5 \%$ of the original yeast, and $0.5 \mathrm{~g}$ of the earth corresponded to $0.0715 \mathrm{~g}$ of adsorbed organic matter.

c) Neutral fatty oil from soy-bean oil :- This was made by dissolving soy-bean oil in a mixture of ether and alcohol $(1: 1)$ and by treating it with about $5 \%$ in excess of the amount of alcoholic $\mathrm{KOH}$ needed to neutralize the free acids in the oil. The mixture was occasionally shaken and allowed to stand for some hours at room temperature, then it was diluted with distilled water and extracted several times with ether. The ethereal solution was washed with distilled water until the filtrate showed no more alkaline reacfion. After dehydrating with anhydrous $\mathrm{Na}_{2} \mathrm{SO}_{4}$, the solution was evaporated as completely as possible in a high vacuum in $\mathrm{CO}_{2}$ atmosphere.

The neutral oil thus obtained consisted of some palmitin and stearin, most of the liquid fatty glycerides of soy-bean oil and also a very little amount of unsaponifiable substances. The saponification and iodine values are as follows :-

\begin{tabular}{l|c|c|c}
\hline Oil & Acid value & Saponif, value & Iodine value \\
\hline Original soy-bean oil & 0.479 & 193 & 139 (Wijs) \\
Neutral fatty oil & - & 196 & 139 (Wijs) \\
\hline
\end{tabular}

d) Saponification of cod-liver oil :- Fifty grams of cod-liver oil were introduced under constant stirring into $125 \mathrm{c.c}$ of $20 \%$ methyl alcoholic $\mathrm{KOH}$ solution and left overnight at room temperature, then the hydrolyzed product was added into the alcoholic solution containing the calculated amount of $\mathrm{CaCl}_{2}$ in order to convert the $\mathrm{K}$-soap into Ca-soap. The precipitate was decomposed with dilute $\mathrm{H}_{2} \mathrm{SO}_{4}$, and the separated fatty acids were extracted with ether. The subsequent procedure was the same as described in the part of the neutral fatty oil from soy-bean oil. Thus a pale yellowish semisolid substance having an indine value of 129 was obtained. 
e) Rice-bran and chrysalis oils. $†$

\begin{tabular}{c|c|c}
\hline \hline Oil & Saponif, value & Iodine value \\
\hline Rice-bran oil & 185 & 107 (Wijs) \\
Chrysalis oil & 194 & 115 (Wijs) \\
\hline
\end{tabular}

Tsujimoto $^{(\theta)}$ stated that fatty acids of rice-bran oil consisted of $20 \%$ palmitic acid, $45 \%$ oleic acid and $35 \%$ isolinoleic acid.

The analytical data of chrysalis oil by $\mathrm{Kimura}^{(7)}$ are listed in the following Table :

Table I.

\begin{tabular}{l|c|c|c|c|c|c|c}
\hline $\begin{array}{l}\text { Acid } \\
\text { value }\end{array}$ & $\begin{array}{c}\text { Sap. } \\
\text { value }\end{array}$ & $\begin{array}{c}\text { Iod. } \\
\text { value }\end{array}$ & $\begin{array}{c}\text { Rhodan } \\
\text { value }\end{array}$ & $\begin{array}{c}\text { Unsap. } \\
\text { matter } \\
\%\end{array}$ & \multicolumn{2}{c}{ Total fat $\%$} \\
\hline 1.57 & 191.58 & 141.8 & 93.0 & 0.98 & & 93.08 \\
\hline & & $\begin{array}{c}\text { Saturat. } \\
\text { acids } \%\end{array}$ & Unsaturated acids $\%$ \\
\hline & & 23.9 & $\begin{array}{c}\text { Oleic } \\
\text { acid }\end{array}$ & $\begin{array}{c}\text { Linoleic } \\
\text { acid }\end{array}$ & $\begin{array}{c}\text { Linolenic } \\
\text { acid }\end{array}$ \\
\hline
\end{tabular}

Feeding of Animals.

Male albino rats of $40 \sim 50 \mathrm{~g}$ were kept two or three in a cage with the various diets listed in Table II. All the diets were supplemented with the known necessary factors as follows: Vitamin A, D and B were supplied by administering biosterol*, irradiated ergosterol* and alcoholic extract of yeast or whole dried yeast respectively. Semi-solid oils were given by dissolving them in liquid paraffin; otherwise, the technique of feeding was the same as in the previous experiments. ${ }^{(1)}$

Table II. Composition (in g) of diets.

\begin{tabular}{|c|c|c|c|c|c|c|c|c|c|}
\hline Diet & $\begin{array}{c}\text { I } \\
\text { (Fat-free diet) }\end{array}$ & II & III & IV & $\mathrm{V}$ & VI & VII & VIII & $\mathrm{JX}$ \\
\hline Casein (fat-free) & 20 & 20 & 20 & 18 & 18 & 18 & 18 & 20 & 20 \\
\hline Potato-starch (fat-free) & 76 & & & & & & & 75 & \\
\hline McCollum salt mixture ${ }^{(8)}$ & 4 & 4 & 4 & 4 & 4 & 4 & 4 & 4 & 4 \\
\hline Polished-rice powder & & 76 & & 75 & 75 & & & & 75 \\
\hline Rice-starch & & & 76 & & & & & & \\
\hline Rice-bran & & & & 3 & & & & & \\
\hline Whole dried yeast & & & & & 3 & & & & \\
\hline $\begin{array}{l}\text { Half-polished rice, retain- } \\
\text { ing about } 40 \% \text { germ. }\end{array}$ & & & & & & 78 & & & \\
\hline Unpolished rice & & & & & & & 78 & & \\
\hline Lecithin & & & & & & & & 1 & 1 \\
\hline
\end{tabular}

I wish to thank Mr. Y. Kawakami of the Kao-Soap Company for funishing the oils.

I wish to thank Dr. M. Sumi and Dr. J. Nakamiya for supplying the irradialed ergosterol and biosterol. 


\section{Results and Discussion.}

a) The growth of rats receiving the polished rice (II) or the rice-starch diet (III) was very much inferior to those receiving the potato-starch diet (I). To our surprise the growth on the two former diets was retarded from the beginning, and the fur and the fore paws were mostly stained with red pigments like blood, and the abnormal condition of fur, giving an appearance of cotton, was often noticed (one of examples being illustrated by Photo.).

Following the bareness around the nose, the mouth and the eyes, there was a tendency to lose the hair on the whole body. The bald areas looked moist and inflamed, but showed no definite characteristic symptoms developed with the fat-free diet, namely the scurfy condition of the skin, "scaliness" of the feet and etc.

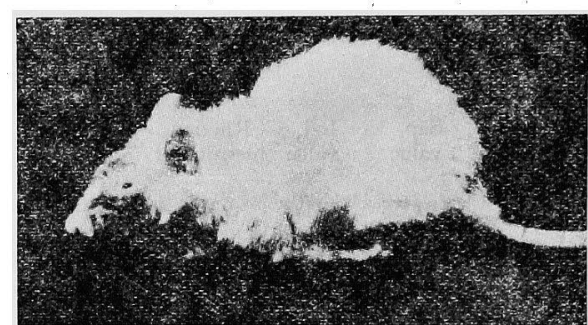

Photo.-Showing the onset of a characteristic symptom of a rat which had been fed with the polished-rice diet (Diet II).

The sick animals on these rice diets could not be recovered in spite of the increased use of the yeast extract, or cured by linoleic acid* unless the rice in the diets was replaced by potato-starch or the yeast extract by whole dried yeast. This fact called our attention to the possibility of the presence of toxic substance or substances in rice, which may be neutralized by whole dried yeast, because the substitution of it for the yeast extract brought about a marked improvement, showing resumption of weight and soft fine hair on the denuded areas.

It was an outstanding phenomenon that rats receiving the polished-rice diet in which $3 \%$ of rice-bran (IV) were added or those receiving the halfpolished-rice diet (VI) developed the symptom resembling that on the polished-rice diet (II), though in the case with the half-polished-rice diet (VI) the rats showed very satisfactory growth (Charts $1 \sim 3$ ). The rats receiving the unpolished-rice diet (VII) attained a normal growth and appearance (Chart 4).

On autopsy of the rats fed with the rice diets, some lesion was often noticed in the liver and the kidney, sometimes exhibiting a white kidney.

b) Linoleic acid with vitamin $\mathrm{B}_{2}$ :- An attempt was made to determine whether linoleic acid could spare vitamin $B_{1}$, as a parallel experiment on vitamin $\mathrm{B}_{2}{ }^{(1)}$ For this purpose the "activated" fuller's earth described previously in this paper was used as vitamin $B_{2}$ source.

* I desire to thank Dr. Y. Sahashi for generously supplylng pure linoleic acid. 
When fed with ration containing $0.5 \%$ of the "activated" fuller's earth, there appeared decline of weight as well as anorexia, and some died in spite of increase of the dose. Since the failure of growth and death were found due to an inadequate technique for the preparation of vitamin $B_{2}$, an autoclaved yeast extract ${ }^{(9)}$ was employed instead of the "activated" fuller's earth; then there was a satisfactory growth for some intervals, but gradually the growth was impaired without any onset of the characteristic convulsion of vitamin $B_{1}$ deficiency. In this case, the administration of 2 drops daily of active oryzanin* induced a remarkable growth on the rats (Chart 5).

This evidence, therefore, led to conclude that linoleic acid had no "sparing" action on vitamin $B_{1}$ as in the case with $B_{2 \cdot}{ }^{(2)}$

c) Lecithin :- The rats reared on the diet containing $1 \%$ lecithin manifested an optimum growth; the fur was very fine and lustrous. In 1932, Trautman $^{(10)}$ stated that the feeding of $2 \mathrm{~g}$ daily of lecithin to a guinea pig accelerated growth, while $4 \mathrm{~g}$ retarded it. In this experiment, when given in the level of $1.5 \%$ of lecithin, growth was definitely improved but a proportion higher than $1.5 \%$ was not used since the pure lecithin was insufficient to ascertain Trautmann's experiment (Chart 6).

d) Neutral fatty oil of soy-bean oil :- Drummond and Gregory ${ }^{(9 ;}$ have reported that the cause of the failure of the rats on the dietaries containing synthetic fat was due to a toxic substance, which was probably produced during the prolonged heating necessary for the esterification. This suggestion led the author to use the natural fatty oil free from fatty acids.

When 3 drops daily of the fatty oil were given the rats grew as healthy as those with 2 drops daily of linoleic acid itself. The oil had an effectiveness in alleviating the deficiency disease produced by the fat-free diet when the symptoms were not too advanced. It was, however, found that its curing power was less than that of linoleic acid itself (Charts 7 and 10).

e) Fatty acids of cod-liver oil :- As growth had been unsatisfactory on the diet containing $0.5 \%$ of the fatty acids, 2 drops daily of $30 \%$ of the acids dissolved in liquid paraffin were given, which exerted no appreciable influence on growth, and the animals had very similar symptoms described on the fat-free diet. ${ }^{(1)}$ When a larger dose was given there was a tendency to produce diarrhea (Chart 8 ).

f) Chrysalis oil :- The group fed with 2 drops daily of the oil showed an excellent growth with a fine lustrous coating of hair. When the weight had exceeded $200 \mathrm{~g}$ the oil was replaced by 2 drops of $30 \%$ rice-bran oil dissolved in liquid paraffin, but the animals could continue growth until the

* I am indebted to Dr. S, Odake for supplying the active oryzanin which cured severe antineuritic pigeons with $4 \mathrm{mg}$ daily. 
experiment was terminated (Chart 9 A).

g) Rice-bran oil :- The feeding was started with the diet containing $1 \%$ rice-bran oil, but the animals soon lost their appetite and began to decline the weight, and there appeared the loss of hair around the nose and eyes. Though administered per os 2 drops daily of $30 \%$ oil dissolved in liquid paraffin or of chrysalis oll, no appreciable influence was exhibited either on growth or on malnutrition. When this condition continued, the animals have become emaciated and been unable to be relieved even though linoleic acid was administered, with the exception of one rat (Chart $9 \mathrm{~B}$ ).

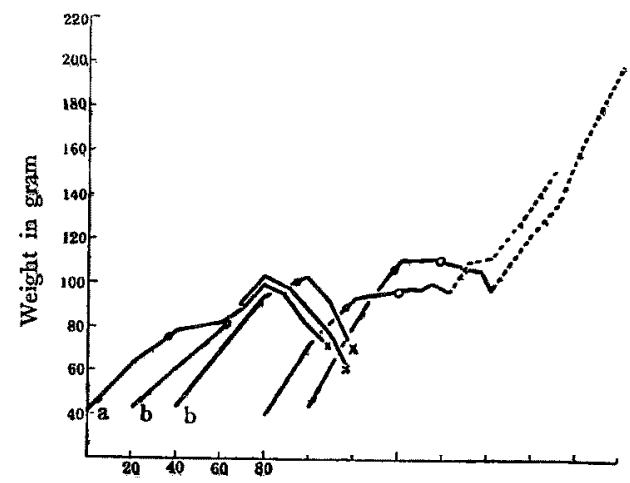

Chart 1.-Curves showing the growth of rats on Diet II before and after the substitution of whole dried yeast for the yeast extract, with administration of 2 drops daily of linoleic acid (a) or wilhout the acid (b). The dotted line indicates the replacement of the yeast extract by whole dried yeast (Diet V), representing the prompt recovering of symptoms and resuming of weight. The small spot indicates an occurrence of bleeding and the small circle shows the sign ef losing hair on the body; $x$ indicates death.

Chart 2.-Curves showing the growth of rats on Diet IV without administration of linoleic acid. During the period $\Delta-\Delta$ the yeast extract was furnished, and thereafter $3 \%$ whole dried yeast replaced it and at the same time the rice-bran was withdrawn. The small spot indicates an occurrence of bleeding.

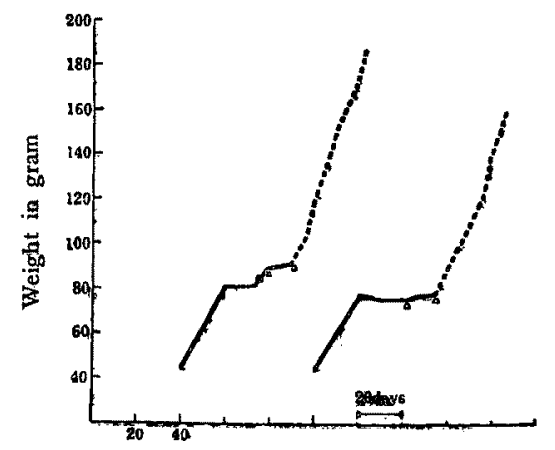




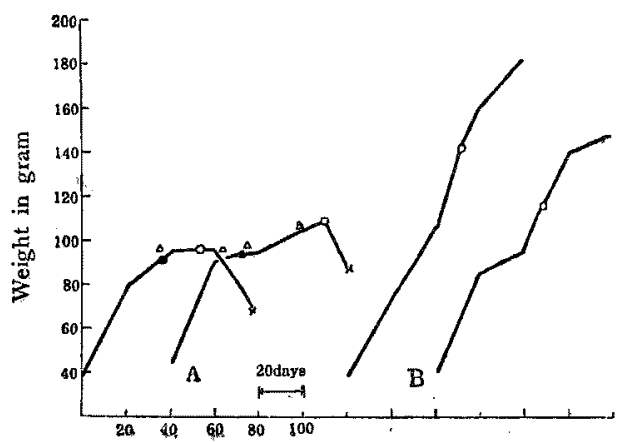

Chart 3.-Curves A showing the growth of rats an Diet III, supplemented with the yeast extract, but without linoleic acid. During the period $\Delta-\Delta$ a larger Jevel of the yeast extract was employed, but showed no appreciable influence on growth. The small spot indicates an occurrence of bleeding and the small circle shows sign of bareness on the body; $x$ indicates death.

Curves B showing the growth of rats on Diet VI without linoleic acid as well as the yeast extract. The small circle indicates an occurrence of losing hair on the body.

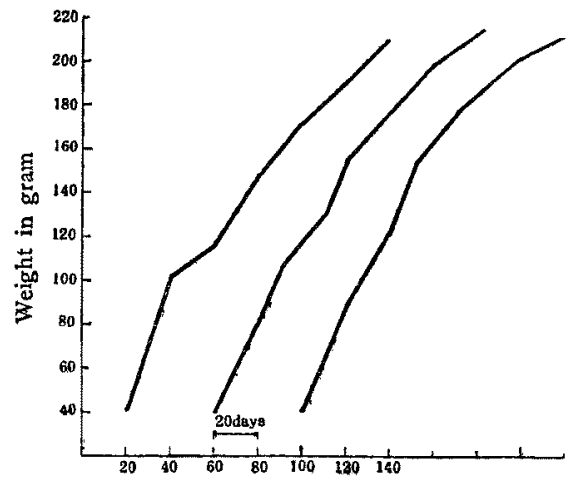

Chart 4.-Curves showing the growth of rats on Diet VII, without linoleic acid as well as the yeast extract. 


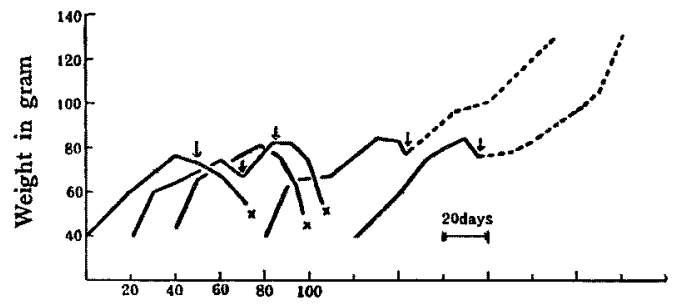

Chart 5.-Curves showing the growth of rats on Diet I, supplemented with "activated" fuller's earth or autoclaved yeast extract as vitamin $\mathbf{B}_{2}$ source, and the renewed growth by supplying 2 drops daily of oryzanin solution as vitamin $B_{1}$. The arrow indicates adding of oryzanin solution; $x$ indicates death.

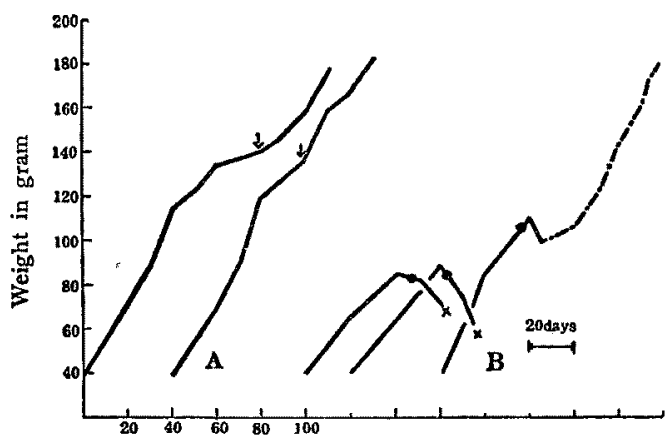

Chart 6.-Curves A showing the growth of rats on Diet VIII, The arrow indscates increase of lecithin to the level of $1.5 \%$.

Curves B showing the growth of rats on Diet IX, and with the replacement of Diet VIII there appears the renewed growth and recovery from symytoms. The small spot indicates an occurrence of bleeding; $x$ indicates death. 


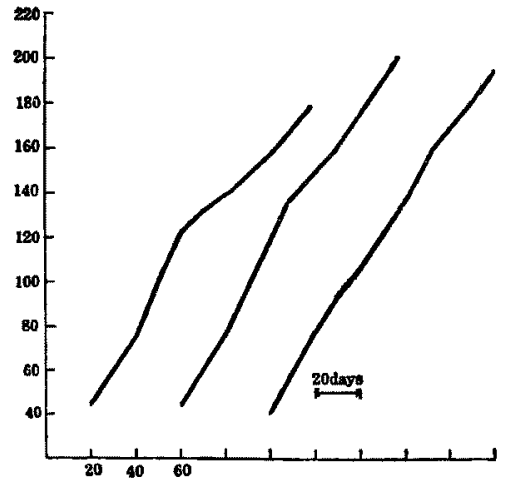

Chart 7.-Curves showing the growth of rats on Diet I, administered with 3 drops daily of neutral fatty oil of soy-bean vil.

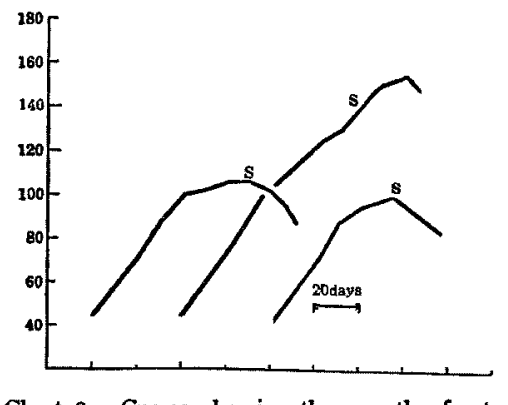

Chart 8.-Curves showing the growth of rats on Diet I, supplemented with 2 drops daily of fatty acids of cudliver oil'; s indicates "scaly" feet condition.

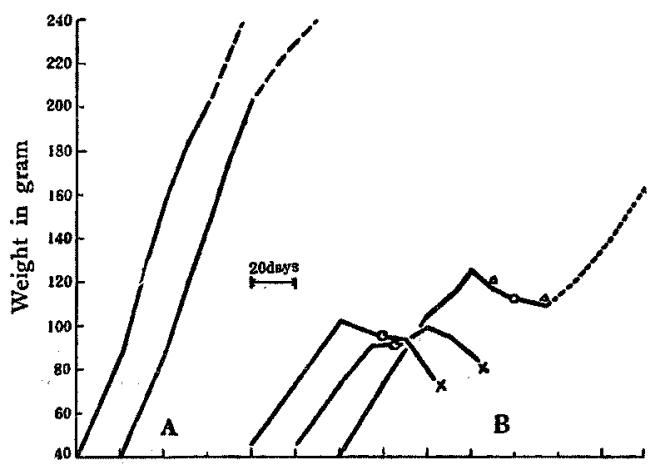

Chart 9.-Curves A showing the growth of rats on Diet I, administered with 2 drops daily of chrysalis oil. The broken line indicates the substitution of rice-bran oil for chrysalis oil.

Curves $B$ showing the growth of rats Diet $I$, supplemented daily with 2 drops of rice-bran oil. During the period $\Delta-\Delta$ the oil was replaced by chrysalis oil. The dotted line shows the renewed growth and recovery from symptoms with administration daily of 2 drops of linoleic acid. The small circle indicates an occurrence of losing hair on the body; $x$ indicates death. 


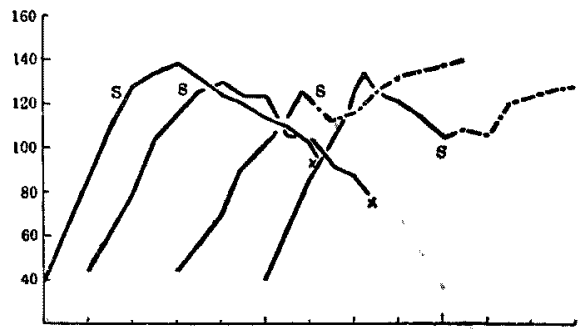

Chart 10.-Curves showing the growth of rats on Diet $I$, and renewed growth and slow recovery from the deficiency disease by treatment with 3 drops daily of fatty oil of soy-bean oil; s indicates "scaly" feet condition; $x$ indicates death.

\section{Summary.}

(1) Growth and the general condition of animals are not attained by the diets containing $76 \sim 78 \%$ of polished-rice powder, rice-starch, or halfpolished rice as carbohydrates, except unpolished-rice diet, though an adequate amount of alcoholic yeast extract is provided. When, however, the yeast extract is replaced by whole dried yeast there appears a prompt recovery of the abnormal condition. The most characteristic phenomena are the occurrence of bareness on the body and of bleeding around the nose, the mouth, and the fore paws.

(2) Lecithin and soy-bean oil possess an almost equal nutritive value in animal physiology to linoleic acid.

(3) Chrysalis oil induces an excellent growth in rats, owing to its content of unsaturated fatty acids, namely linoleic and linolenic acids.

(4) The abnormal condition in nutrition of the rats fed on the ricebran oil is not improved by administration of either chrysalis oil or linoleic acid. The cause of malnutrition and of the failure of growth with the former oil is not yet clear.

(5) The results of experiments with fatty acids prepared from cod-liver oil show that they cannot maintain the growth of animals and that the large dose causes a disturbance of the gastro-intestine.

(6) Linoleic acid is unable to "spare" either vitamin $B_{1}$ or $B_{2}$.

The author wishes to express her deep gratitude to Professor U. Suzuki for his many helpful suggestions throughout this work. She is indebted to the Keimei Society for financial assistance and is also grateful to Miss K. Miti for her willing help in feeding of the animals and preparing of the diets. 


\section{Literature}

(1) U. Tange: Sc. Pap, I. C. P. R., 20, 13 (1932).

(2) H. M. Evans and S. Lepkovsky: J. Biol. Chem., 96, 143 (1932).

(3) V. Reader: Biochem. J., 23, 689 (1929) ; 24, 77, 1827. (1930, 1-2).

(4) H. Chick and A. M. Copping: Biochem. J., 24, 1764 (1930).

(5) B. T. Narayanan and J. C. Drummond; Biochem. J., 24, 9 (1930).

(6) M. Tsujimoto: "Kögyö-Kagaku-Zasshi", 12, 297 (1909).

( 7 ) W. Kimura: "Kögyō-Kagaku-Zasshi”, 33, 760 (1930).

(8) E. V. McCollum and N. Simmonds: J. Biol. Chem., 33, 63 (19i8).

(9) E. Gregory und J. C. Drummond: Zeit. f. Vitaminforschung B, und 1-Hef t 4, 273 (1932).

(10) A. Trautmann: Chem. Abst, 26, 4087 (1932), 